\title{
Relationship between internal accuracy and load-bearing capacity of minimally invasive lithium disilicate occlusal veneers
}

Mörikofer, Nina ; Benić, G I ; Park, J M ; Özcan, Mutlu ; Hüsler, Jürg ; Ioannidis, Alexandros S

\begin{abstract}
Purpose: To test whether internal accuracy affects the load-bearing capacity of 0.5-mm-thick occlusal veneers made out of milled or heat-pressed lithium disilicate (LS2). Materials and methods: Extracted human molars $(\mathrm{N}=80)$ were divided into four groups $(\mathrm{n}=20$ each) depending on the bonding substrate (enamel $[\mathrm{E}]$ or dentin $[\mathrm{D}]$ ) and the fabrication method (milling [CAD] or heat pressing [PRE]) for the occlusal LS2 veneers: (1) E-CAD, (2) D-CAD, (3) E-PRE, or (4) D-PRE. After restoration fabrication, the abutment teeth and the corresponding restorations were scanned and superimposed in order to measure the marginal and internal accuracy. After adhesive cementation, the specimens were thermomechanically aged and thereafter loaded until fracture. The load-bearing capacities (Fmax) were measured. Fmax and the marginal and internal accuracy between the groups were compared using Kruskal-Wallis test $(\mathrm{P}<.05)$ and pairwise group comparisons. In addition, the relationship between Fmax and the internal accuracy was analyzed using Spearman rank correlation. Results: Median Fmax values (and first and third quartiles) per group were as follows: 1,495 N (Q1: 932; Q3: 2'318) for E-CAD; 1,575 N (Q1: 1,314; Q3: 1,668) for E-PRE; 1,856 N (Q1: 1,555; Q3: 2,013) for D-CAD; and 1,877 N (Q1: 1,566; Q3: 2,131) for D-PRE. No statistical difference was found between the groups $(\mathrm{P}=.0981)$. Overall, the internal accuracy in the areas of the cusp $(\mathrm{P}<.0007)$ and fossa $(\mathrm{P}<.0001)$ showed significant differences. While no significant differences were detected in the marginal area $(\mathrm{P}=.3518)$, a significant correlation with a negative linear relationship was found between the 3D internal accuracy and the Fmax values $(\mathrm{P}=.0007)$. Conclusion: An increase in the internal accuracy raised the load-bearing capacity of minimally invasive LS2 occlusal veneers. In general, the restorations bonded to dentin in the occlusal regions showed a better accuracy compared to those bonded to enamel.
\end{abstract}

DOI: https://doi.org/10.11607/ijp.6735

Posted at the Zurich Open Repository and Archive, University of Zurich

ZORA URL: https://doi.org/10.5167/uzh-203396

Journal Article

Accepted Version

Originally published at:

Mörikofer, Nina; Benić, G I; Park, J M; Özcan, Mutlu; Hüsler, Jürg; Ioannidis, Alexandros S (2021). Relationship between internal accuracy and load-bearing capacity of minimally invasive lithium disilicate occlusal veneers. International Journal of Prosthodontics, 34(4):365-372.

DOI: https://doi.org/10.11607/ijp.6735 
Relationship between internal accuracy and load-bearing capacity of minimally invasive lithium disilicate occlusal veneers

Mörikofer $\mathrm{N}^{\mathrm{a}}$, Benic $\mathrm{Gl}{ }^{\mathrm{b}}$, Park $\mathrm{JM}^{\mathrm{c}}$, Özcan $\mathrm{M}^{\mathrm{b}}$, Hüsler $\mathrm{J}^{\mathrm{d}}$, loannidis $\mathrm{A}^{\mathrm{b}}$

Key words: lithium disilicate ceramic, occlusal dental veneers, load-bearing capacity

Address for correspondence:

Dr. med. dent. Alexis loannidis

Clinic of Reconstructive Dentistry

Center of Dental Medicine, University of Zurich

Plattenstrasse 11

$\mathrm{CH}-8032$ Zurich, Switzerland

Phone: +41446340404

Fax: +41446344305

E-Mail: alexis.ioannidis@zzm.uzh.ch

${ }^{a}$ Doctoral student, Clinic of Reconstructive Dentistry, Center of Dental Medicine, University of Zurich, Zurich, Switzerland

${ }^{\mathrm{b}}$ Clinic of Reconstructive Dentistry, Center of Dental Medicine, University of Zurich, Zurich, Switzerland

${ }^{c}$ Department of Prosthodontics, Yonsey University, College of Dentistry, Seoul, Korea

${ }^{\mathrm{d}}$ Department of Mathematical Statistics, University of Bern, Switzerland 


\section{Abstract}

Objectives: To test whether the internal accuracy affects the load-bearing capacity of $0.5 \mathrm{~mm}$-thick occlusal veneers made out of milled or heat-pressed lithium disilicate (LS2).

Material and methods: Extracted human molars $(N=80)$ were divided into 4 groups $(n=20)$ depending on the bonding substrate (enamel or dentin) and the fabrication method (milling or heatpressing) for the $0.5 \mathrm{~mm}$ thick occlusal lithium disilicate (LS2) veneers: milling, bonding to enamel (ECAD) or dentin (D-CAD); fabricated by heat-pressing and bonded to enamel (E-PRE) or dentin (DPRE). After restoration fabrication, the abutment teeth and the corresponding restorations were scanned and superimposed in order to measure the marginal and internal accuracy. After adhesive cementation, the specimens were thermo-mechanically aged and thereafter loaded until fracture. The load-bearing capacities $\left(F_{\max }\right)$ were measured. $F_{\max }$ and the marginal and internal accuracy between the groups were compared using the Kruskal-Wallis test $(p<0.05)$ and pairwise group comparison. In addition, the relationship between $F_{\max }$ and the internal accuracy was analyzed using the Spearman rank correlation.

Results: Median $F_{\max }$ values (and first and third quartiles) were as follows per group: 1'495 N (Q1: 932; Q3: 2'318) for E-CAD, 1'575 N (Q1: 1'314; Q3: 1'668) for E-PRE, 1'856 N (Q1: 1'555; Q3: 2'013) for D-CAD and 1'877 N (Q1: 1'566; Q3: 2'131) for D-PRE. No statistical difference was found between the groups $(p=0.0981)$. Overall, the internal accuracy in the areas of the cusp $(p<0.0007)$ and fossa $(p<0.0001)$ showed significant differences. While no significant differences were detected in the marginal area $(p=0.3518)$, a significant correlation with a negative linear relationship was found between the 3D-internal accuracy and the $F_{\max }$ values $(p=0.0007)$.

Conclusions: An increase in the internal accuracy raised the load-bearing capacity of minimally invasive lithium disilicate occlusal veneers. In general, in the occlusal regions the restorations bonded to dentin showed a better accuracy compared to those bonded to enamel. 


\section{Introduction}

For the treatment of a worn dentition, indirect minimally invasive restorative concepts can be indicated (1). Occlusal veneers are a suitable restorative treatment option for the posterior dentition for this indication. For these restorations, presently an enormous amount of different reconstructive materials are available on the market. They include resin-based materials, polymer-infiltrated ceramic network materials (PICN), reinforced ceramics or/and high-strength ceramics. Resin-based materials and PICN come along with a low hardness and brittleness compared to ceramic materials and thus may offer favourable properties when applied to reduced thicknesses (2). On the other hand, the high flexural strength and fracture toughness of reinforced glass ceramics or high strength ceramics could be in favour towards this application $(\underline{3}, \underline{4})$. Lithium disilicate (LS2) - as a representative of the reinforced glass ceramics - shows favourable mechanical and bonding properties and can be used for the fabrication of these occlusal veneers $(\underline{5-8})$. LS2 restorations can either be manufactured by heatpressing or by CAD/CAM procedures - namely milling ( $\underline{9})$. The fabrication procedures itself has an impact on the internal accuracy of the restoration (10). The internal accuracy describes the distance between the inner surface of the restoration and the underlying tooth and thus determines the thickness of the cement layer. During fabrication, its dimension is determined by the thickness of the spacer. For pressed LS2 restorations - being manufactured with the conventional lost-wax and heat-press technique - the spacer-thickness is defined by a paint-on die spacer (11). In CAD/CAM technologies however, the spacer-thickness can be predefined during the design procedure of the restoration. This parameter has been addressed to influence the performance of all-ceramic restorations $(\underline{12-14})$. The interface-zone between the restoration and the tooth has been accounted as the zone of crack initiation - especially in thin restorations (12). The initiated cracks can propagate towards the occlusal surface or the margin of the restoration and finally lead to catastrophic failure of the restoration (12). Thus, the mechanical stability of cemented restorations may depend on the dimensions of this zone and may decrease with increasing thickness. Besides that, the manufacturing-method of LS2 restorations can affect the mechanical resistance to fractures directly, (9) as pressed and CAD/CAM fabricated 
restorations does not have the same physical properties $(\underline{9})$. With regard to their fracture toughness, pressed restorations seem to perform superior compared to those which are CAD/CAM fabricated (9). To summarize, the selection of the material with the associated manufacturing procedure and the dimension of the spacer-thickness can have an influence on the resistance to fractures of restoration. Thus, the appropriate material selection is crucial when restoring worn teeth with ultra-thin occlusal veneers. However, little is known about the influence of these parameters on the mechanical performance of minimally invasive restorations in the load-bearing jaw regions. Furthermore, the substrate which is present for the bonding of the restoration plays a pivotal role. Therefore, the aim of the present study was to test whether the load-bearing capacity of ultra-thin occlusal veneers, made either out of pressed or milled LS2 ceramic, is influenced by their internal accuracy and the used bonding substrate. The hypothesis was that the load-bearing capacity decreases with increasing dimension of the interface zone in the occlusal area between the restoration and the tooth. 


\section{Material and Methods}

\subsection{Groups}

The investigated groups (Table 1) differed in the tooth substrate on which the occlusal veneers were bonded (E: enamel or D: dentin) and in the manufacturing procedure of the lithium disilicate reinforced glass-ceramic (LS2) restorations (CAD: milling or PRE: heat-pressing). The following four groups $(n=$ 20 per group) were assessed:

(1) "E-CAD": CAD/CAM fabricated LS2 occlusal veneer (IPS e.max CAD, Ivoclar Vivadent, Schaan, Liechtenstein) bonded to enamel;

(2) "E-PRE”: heat-pressed LS2 occlusal veneer (IPS e.max press; Ivoclar Vivadent) bonded to enamel;

(3) "D-CAD": CAD/CAM fabricated LS2 (IPS e.max CAD, Ivoclar Vivadent, Schaan, Liechtenstein) bonded to dentin;

(4) “D-PRE": heat-pressed LS2 occlusal veneer (IPS e.max press; Ivoclar Vivadent) bonded to dentin.

\subsection{Specimen preparation}

80 extracted intact human molars were collected and embedded in a self-curing resin (Technovit 4071; Kulzer, Wasserburg, Germany) with the apical part facing downwards in an acrylic hollow cylinder (Figure 1). To simulate tooth wear caused by attrition, in 40 randomly selected specimens, the occlusal enamel was removed without extending into the dentin. For the other 40 specimens, defects with exposed dentin were prepared. For this purpose, the occlusal enamel was completely removed until only a border of enamel remained and the central part contained solely of dentin. In addition, in all 80 specimens, sharp edges were rounded off and if present, fissures were slightly opened with a diamond bur under water cooling. During the entire study procedures, the specimens were stored in a solution of $0.5 \%$ chloramine $\mathrm{T}$ and distilled water. 


\subsection{Scanning procedures, restoration design and fabrication}

Digital impressions of the prepared teeth were performed by means of an intraoral scanner (Cerec Omnicam; Software-Version 4.4, Sirona, Bensheim, Germany). Subsequently, the scans were transferred to a design software (InLab; Sirona) to design an occlusal restoration with a standardized thickness of $0.5 \mathrm{~mm}$ (range $0.3-0.7 \mathrm{~mm}$ ) for each specimen.

The specimens of the groups E-CAD and D-CAD were directly milled out of pre-fabricated LS2 ceramic ingots (IPS e.max CAD; Ivoclar Vivadent) using a 5-axis milling machine (MC X5; Sirona).

For the specimens of the groups E-PRE and D-PRE, first, an acrylic polymer template (Vita CAD Waxx, Vita Zahnfabrik, Bad Säckingen, Germany) was produced by milling procedures (MC X5; Sirona). Consecutively, the template was used to produce heat-pressed LS2 restorations (IPS e.max Press; Ivoclar Vivadent) by applying the "lost-wax and press-technique". Therefore, the template was vested (IPS PressVest Premium; Ivoclar Vivadent) and heated to complete dissolution. Thereafter, the LS2 ingot was heat pressed into the resulting space and carefully devested after cooling.

\subsection{Evaluation of the marginal and internal adaption}

To measure the internal adaption before cementing the restorations, an additional scanning procedure was performed. The three scans of the preparation, restoration, and the relationship between the two after setting were acquired with an intraoral scanner (3Shape Trios; 3Shape, Copenhagen, Denmark) and superimposition protocol was followed by triple-scan method introduced from several researches (15-17). The prepared teeth were scanned and the restorations were scanned with a reference structure on the occlusal surface. A third scan was taken in the occlusion mode with the restoration in place. Standard tessellation (STL) files were created and imported into a software for 3D analysis (Geomatic verify 64Bit, ver. 2015.2.0; 3D Systems, Rock Hill, SC, US). The alignment between the preparation and restoration was checked by matching the reference cubic structure with two cylinders at a right angle, which acted as the fiducial marker. This was conducted by the ' $\mathrm{N}$ point align transform', and 'Best fit alignment' command of the inspection software. The internal and the marginal adaptions were measured in the mesio-distal $(\mathrm{MD})$ and the bucco-lingual $(\mathrm{BL})$ cross-sections at different points: 
2× margin $\mathrm{MD}, 2 \times$ margin $\mathrm{BL}, 2 \times$ cusp $\mathrm{MD}, 2 \times$ cusp $\mathrm{BL}, 1 \times$ fossa $\mathrm{MD}, 1 \times$ fossa $\mathrm{BL}$ (Figure 2). For further analysis, the measurements were pooled to margin (mean of measurements margin MD, margin $B L$ ), cusp (means of measurements cusp MD, cusp BL) and fossa (mean of measurements fossa MD, fossa $\mathrm{BL}$ ). In addition, color maps of the 3-dimensionally (3D) assessed internal adaption were generated and the total surface between the inner surface of the restoration and the abutment tooth was measured. This 3D-internal adaption was further used for the correlation analysis to the loadbearing capacity.

\subsection{Cementation protocols}

The cementation protocols varied within the two different tooth substrate groups (enamel or dentin) and followed the manufacturer's recommendations. The restorations were etched for $20 \mathrm{~s}$ with $5 \%$ hydrofluoric acid (HF; Ivoclar Vivadent) before water-spraying and air-drying. A silane was applied for $60 \mathrm{~s}$ (Monobond Plus; Ivoclar Vivadent) and gently air-dried. Enamel and dentin were etched $30 \mathrm{~s}$ with $35 \%$ phosphoric acid (Ultraetch; Ultradent, Utah, USA) and subsequently sprayed with water before gently air-drying. In the groups with exposed dentin, latter was additionally conditioned (Syntac Primer/Syntac Adhesive; Ivoclar Vivadent). Thereafter, a bonding agent was applied on the prepared teeth and on to the restorations (Heliobond; Ivoclar Vivadent) before gently air-dried.

After conditioning, the restorations were adhesively bonded to the prepared teeth, using a dual-curing resin cement (Variolink Esthetic; Ivoclar Vivadent). The excess cement was carefully removed before light-curing for 6 x $40 \mathrm{~s}$ was performed.

\subsection{Aging procedures}

Thermo-mechanical aging was applied to the specimens, using a custom-made chewing simulator (18). The aging parameters were set as follows: thermo-cycling with $5-50^{\circ} \mathrm{C}$ and a dwelling time of $120 \mathrm{~s}$; chewing simulation with 1'200'000 cycles of $49 \mathrm{~N}$ force at $1.67 \mathrm{~Hz}$ loading frequency. A vertical indenter with a rounded tip of $\varnothing 8 \mathrm{~mm}$ and made of corrosion-free steel, was used to load the specimens in axial direction to the occlusal plane. 


\subsection{Static loading}

After ensuring the integrity of the specimens under a stereomicroscope (magnification 1.25x), the specimens were further tested in an universal testing machine (Zwick / Roell Z010; Zwick, Ulm, Germany). In order to test the static fracture load, an indenter hit the occlusal surface of the specimen in a perpendicular direction (crosshead speed $1 \mathrm{~mm} / \mathrm{min}$ ) until the specimen fractured. $F_{\text {initial, }}$, being the force needed to form the first crack, while $F_{\max }$ is the ultimate force to completely fracture the restoration. On digital photographs and with loups (magnification $2.5 \times$ ) the types of failure were identified and scored into Score $0=$ no visible fracture; Score $1=$ cohesive fracture within the restoration; Score 2 = cohesive fracture of the restoration and of the cement layer; Score $3=$ fracture of the restoration-cement-tooth complex.

\subsection{Statistical analysis}

The metric variables with mean, median, standard deviations, quartiles, minimum and maximum were described. Categorical variables were summarized by counts and proportions of the categories. The comparisons of the group medians of the metric variables were performed with nonparametric methods (Kruskal-Wallis test). Multiple comparisons of two groups are based on adjusted p-values, using Dwass-Steel-Fligner method (DSF). The proportions of the categorical parameters with the chi-squares test were compared (exact $p$-values derived). P-values $<0.05$ were considered to be statistically significant in all tests. 


\section{Results}

\subsection{Fatigue resistance}

All tested specimens survived the thermo-mechanical aging procedures without any fracture.

\subsection{Load-bearing capacity}

The median $F_{\text {initial }}$ values and their first (Q1) and third (Q3) interquartile amounted for E-CAD $845 \mathrm{~N}$ (Q1: 600; Q3: 1'500), for E-PRE 700 N (Q1: 610; Q3: 1'050), for D-CAD 1'400 N (Q1: 900; Q3: 1'500) and for D-PRE 1'100 N (Q1: 900; Q3: 1'650) (Table 2, Figure 3). Comparing the groups under investigation, significant differences $(K W: p=0.0248)$ were only found between E-PRE and D-PRE ( $p$ $=0.0169)$.

The following median $F_{\max }$ values and their respective Q1 and Q3 were found: 1'495 N (Q1: 932; Q3: 2'318) for E-CAD, 1'575 N (Q1: 1'314; Q3: 1'668) for E-PRE, 1'856 N (Q1: 1'555; Q3: 2'013) for DCAD and 1'877 N (Q1: 1'566; Q3: 2'131) for D-PRE (Table 2, Figure 4). No statistically significant differences were found comparing the tested groups $(K W: p=0.0981)$.

\subsection{Failure types}

The Chi-squares tests comparing the failure types of all groups (Table 3) showed no statistically significant differences $(p=0.0715)$.

\subsection{Marginal and internal adaption}

The internal and the marginal adaption (Table 4) was measured in different cross-sections and at different locations: margin, cusp, fossa. In the area of the margin, medians and respective Q1 and Q3 values of $115 \mu \mathrm{m}$ (Q1: 83; Q3: 167) were found for group E-CAD, $113 \mu \mathrm{m}(\mathrm{Q} 1: 90 ; \mathrm{Q3}$ : 137) for EPRE, $107 \mu \mathrm{m}$ (Q1: 86; Q3: 118) for D-CAD and $93 \mu \mathrm{m}$ (Q1: 75; Q3: 120) for D-PRE. The medians in the area of the margins showed no significant differences between the groups (KW: $p=0.3518$ ). 
In the area of the cusp, the following medians and respective Q1 and Q3 were measured: $229 \mu \mathrm{m}$ (Q1: 177; Q3: 285) for E-CAD, $239 \mu \mathrm{m}$ (Q1: 205; Q3: 315) for E-PRE, $185 \mu \mathrm{m}$ (Q1: 151; Q3: 211) for DCAD and $145 \mu \mathrm{m}$ (Q1: 105; Q3: 177) for D-PRE. Significant differences were found in this area (KW: 0.0007) between E-CAD and D-CAD (DSF: $p=0.0495)$, between E-CAD and D-PRE (DSF: $p=$ 0.0011), between E-PRE and D-CAD (DSF: $p=0.0013)$ and between E-PRE and D-PRE (DSF: $p<$ 0.0001).

The measurements in the area of the fossa revealed median and Q1/Q3 values of $560 \mu \mathrm{m}$ (Q1: 451 Q3: 729) in E-CAD, $628 \mu \mathrm{m}$ (Q1: 541; Q3: 796) for E-PRE, $264 \mu \mathrm{m}$ (Q1: 225; Q3: 334) for D-CAD and $406 \mu \mathrm{m}$ (Q1: 162; Q3: 580) for D-PRE. Significant differences were found also in the area of the fossa $(\mathrm{KW}: \mathrm{p}<0.0001)$ between E-CAD and D-CAD (DSF: $p<0.0001)$, between E-PRE and D-CAD (DSF: $p<0.0001)$ and between E-PRE and D-PRE (DSF: $p=0.0046)$.

The medians and Q1/Q3 values of the 3-dimensionally assessed internal adaption (color map) amounted of $196 \mu \mathrm{m}$ (Q1: 159; Q3: 280) for E-CAD, $214 \mu \mathrm{m}$ (Q1: 171; Q3: 248) for E-PRE, $186 \mu \mathrm{m}$ (Q1: 149; Q3: 200) for D-CAD and $155 \mu \mathrm{m}$ (Q1: 120; Q3: 187) for D-PRE. Comparing the medians of the color map measurements, significant differences were found (KW: $p=0.0007)$ between $E-C A D$ and D-PRE (DSF: $p=0.0172$ ), between E-PRE and D-CAD (DSF: $p=0.0411$ ) and between E-PRE and D-PRE (DSF: $p=0.0019)$.

\subsection{Correlation of internal adaption and load-bearing capacity}

The measurement values of the internal adaption gathered by the color map measurements, were correlated to the $F_{\max }$ values for the load-bearing capacity by means of a Spearman correlation (Figure 5). A significant correlation with a negative linear relationship was found between these data $(p=$ 0.0019). In other words, with the increasing distance between the restoration and the abutment tooth, the capacity to withstand loading forces also increased. 


\section{Discussion}

In the present study it was disclosed that the proximity of ultra-thin restoration made of LS2 to the abutment tooth had a significant correlation to its capacity to withstand occlusal forces. In general, the restorations bonded to dentin showed a higher accuracy in the occlusal areas of the cusps and fossae compared to those bonded to enamel. Neither the fabrication method (milling / heat-pressing) nor the substrate for bonding (enamel / dentin) had an influence on the maximum load-bearing capacity of the occlusal veneers. The hypothesis that the load-bearing capacity decreases with increasing thickness of the interface zone was confirmed in this study. A significant correlation with a negative linear relationship was found between the load-bearing capacity and the internal adaption. To the knowledge of the authors, no previous study investigated this correlation.

No complications or failures in any of the tested groups occurred during the phase of artificial aging. Thus, all materials resisted the thermo-mechanical aging, which simulates dynamic loading forces under clinical conditions. The clinical conditions with temperature changes in the wet oral cavity and dynamic fatigue alterations were imitated by a chewing simulator. The used protocol of aging has been reported to simulate 5 years of clinical service $(\underline{19-21})$.

The hypothesis of the present study was set due to the fact that the cement layer between the restoration and the tooth is contemplated to be responsible for the crack initiation $(\underline{12}, \underline{22})$ and, therefore, the thickness of this zone may influence the load-bearing capacity of ceramic restorations. The thickness of the interface normally depends upon the fabrication method for LS2 $(\underline{23}, \underline{24})$. The internal discrepancies of CAD/CAM fabricated LS2 restorations are known to be significantly higher than in heat-pressed ones $(\underline{23}, \underline{24})$. This has also been shown in a clinical study comparing digital and conventional workflows $(\underline{25})$. In terms of the internal adaption, this previous study showed that LS2 crowns which were fabricated by heat-pressing revealed a higher accuracy than CAD-milled LS2 restorations (25). Accordingly, another clinical study showed a higher accuracy of conventionally fabricated frameworks compared to CAD/CAM fabricated ones (26). This fact can be attributed to the 
shortcomings of CAD/CAM technologies within the milling process. Narrow configurations of the inner surface cannot be accurately milled by rotating instruments. In this context is has to mentioned that in the present investigation the wax-templates for heat-pressing were milled. For the above-mentioned reason, the drawbacks of CAD/CAM fabrication affected the internal discrepancy and consequently, the stability of the heat-pressed restorations. The present findings without differences in the internal adaption between the milled and the heat-pressed restorations reflect these thoughts. Probably on the same grounds, the here revealed results of the internal adaption differed for the restorations fabricated for the enamel compared to the restorations fabricated for the dentin specimens. In the areas of the cusps and the fossae, which reflects the internal adaption, a significantly higher accuracy was found for the dentin specimens. For methodological motives, the occlusal relief of the enamel and of the dentin specimens evidently differed. In order to mimic defects reaching into the dentinal areas, the occlusal relief of the dentin specimens were flat. In contrast, the relief of the enamel specimens were much more pronounced and thus showed more small configurations of the inner surface which might have caused inaccuracy during the milling process.

Typically, if LS2 restorations are bonded to enamel, they reveal a higher fracture resistance compared to the ones bonded to dentinal substrate (27). A previous study showed median fracture resistances of $4^{\prime} 173 \mathrm{~N}$ on enamel, whereas the values amounted to $3^{\prime} 840 \mathrm{~N}$ on dentin (27). This was not the case in the present investigation with $F_{\text {initial }}$ and $F_{\max }$ values tending to be higher in the groups in where the restorations were cemented onto dentin. Two previous studies with very similar study conditions tested heat-pressed LS2 restorations bonded to enamel (ㅁ) and dentin (ㅁ). Similarly to the present investigation, in both studies occlusal veneers of $0.5 \mathrm{~mm}$ thickness were thermo-mechanically aged and consecutively loaded until fracture $(\underline{6}, \underline{7})$. The revealed $F_{\max }$ values reached median load-bearing capacities of 1 '692 (enamel) and 1'165 $\mathrm{N}$ (dentin) $(\underline{6}, \underline{7})$. In contrast to the present investigation, the measurements of the previous studies followed the expected order by having a higher load-bearing capacity measured on enamel than on dentin. Comparing the fracture toughness of the LS2 fabricated by heat-pressing or milling, heat-pressed restorations seem to perform superior compared to those who are CAD/CAM fabricated (9 $)$. Although the chemical composition of these two materials is very 
similar, LS2 for CAD/CAM fabrication has a larger glassy matrix and smaller crystals compared to the material being used for heat-pressing (ㅁ). The differences in the mechanical performance can be attributed to these distinctions. In the present study however, no differences between the fabrication methods were found regarding their load-bearing capacity. In contrast to the measurements of the mechanical performance of a dental material itself, here a complex of tooth-cement-restoration was tested on their mechanical behavior. One can assume that the adhesion between the restoration and the abutment tooth is capable of compensating for the individual inferior mechanical properties of the weaker material. In this context it can be imagined that the morphology of the restoration in connection with the adhesion to the tooth surface may change the classical ranking of the materials regarding their mechanical properties, as shown also in other studies $(\underline{6}, \underline{7})$. Furthermore, as already mentioned above, the higher accuracy of the dentin-bonded restorations might be attributed to the relief differences between enamel and dentin specimens. As found in the present study, the load-bearing capacity depends on this parameter. Presumably, dentin- and enamel-bonded specimens with identical occlusal configurations would follow more the 'classic hierarchy' with enamel-bonded restorations showing a higher load-bearing capacity than dentin-bonded restorations.

In this study, the 'triple-scan method' was used to measure the internal adaptation of restorations (1517). This protocol is a three-dimensional approach to assess the gap between abutment and restoration with three scans: abutment, restoration and restoration on top of the abutment. The three scans were obtained by an intraoral scanner. The accuracy of these scanners has been reported in previous studies (28-31). Their collective evidence is that there may be deviations in the full-arch scan depending on the performance of the scanner system. However, they show a clinically acceptable accuracy in single tooth and quadrant arch scans (31). Here very small objects were scanned, presumably 1.5 to 2 times smaller than the optical window of the intraoral scanner. This reduces the distortion which is a result of the stitching process of multiple three-dimensional scans. Regarding the distortion of the scan, a desktop scanner would have been advantageous. However, undercuts of restorations can be acquired with a higher reliability by means of an intraoral scanner. Usually, scanning of the outer and inner surface of a restoration have to be performed in two scans when a desktop scanner is used. The matching process of these two scans would include a further source for 
error. Furthermore, no powder application was needed by the used intraoral scanner, while desktop scanners usually require the application of a powder which might influence the measurements. The here used method to determine the accuracy, allows an unlimited number of cross-sections and eliminates manual sources of errors that can appear in the classical replica technique (32). An acceptable validity of the measurement method employed in this study has been shown (17).

In clinical cases, the surface of severe erosive or abrasive tooth wear usually leads to the presence of sclerotic dentin. There are structural differences between sclerotic dentin and the freshly cut dentin which was used in the present study. The in vitro simulation of identical sclerotic dentin in a study setup however, is very difficult or impossible to simulate. Nevertheless, for the above-mentioned structural differences, in this present where we could not simulate the sclerotic dentin, the results could reflect only adhesion to freshly cut dentin. However, interestingly enough clinical evidence suggests an acceptable performance over 3.5 years in terms of bonding quality after amalgam replacement ( $\underline{33})$, which may be more critical than expected than bonding to eroded dentin. Furthermore, in the presence of tooth wear, the margins enclose enamel which adds to the durability of adhesion on erosive/sclerotic dentin. 


\section{Conclusions}

This study revealed a significant correlation of the internal adaption to the load-bearing capacity. An increase in the internal accuracy raised the load-bearing capacity of minimally invasive $0.5 \mathrm{~mm}$-thick occlusal veneers made of lithium disilicate reinforced glass-ceramic. In general, the restorations bonded to dentin showed a higher accuracy in the areas of the cusps and fossae compared to those bonded to enamel. In addition, enamel- and dentin-bonded specimens did not differ regarding their load-bearing capacity.

\section{Conflict of Interest}

supported this investigation with a research grant. (\#2017_1a). The authors acknowledge the material support from Ivoclar Vivadent.

\section{Acknowledgements}

The authors thank the dental technician for his help in the fabrication of the restoration specimens and proof reading. 


\section{Tables}

Table 1 - Groups with the given substrate for bonding, the fabrication procedures and the used restorative materials with their respective chemical composition.

\begin{tabular}{|c|c|c|c|}
\hline Group & Substrate for bonding & Fabrication procedure & Restorative material \\
\hline E-CAD & Enamel & CAD/CAM milling & $\begin{array}{l}\text { Lithium disilicate ceramic (IPS e.max CAD; Ivoclar Vivadent, Schaan, Liechtenstein): } \\
\mathrm{SiO}_{2}(57-80 \mathrm{wt} \%), \mathrm{Li}_{2} \mathrm{O}(11-19 \mathrm{wt} \%), \mathrm{K}_{2} \mathrm{O}(0-13 \mathrm{wt} \%), \mathrm{P}_{2} \mathrm{O}_{5}(0-11 \mathrm{wt} \%), \mathrm{ZrO}_{2}(0-8 \\
\mathrm{wt} \%), \mathrm{ZnO}(0-8 \mathrm{wt} \%), \mathrm{AlO}_{3}(0-5 \mathrm{wt} \%), \mathrm{MgO}(0-8 \mathrm{wt} \%)\end{array}$ \\
\hline E-PRE & & Heat-pressing & $\begin{array}{l}\text { Lithium disilicate ceramic (IPS e.max Press; Ivoclar Vivadent): } \\
\mathrm{SiO}_{2}(57-80 \mathrm{wt} \%), \mathrm{Li} \mathrm{i}_{2} \mathrm{O}(11-19 \mathrm{wt} \%), \mathrm{K}_{2} \mathrm{O}(0-13 \mathrm{wt} \%), \mathrm{P}_{2} \mathrm{O}_{5}(0-11 \mathrm{wt} \%), \mathrm{ZrO}_{2}(0-8 \\
\mathrm{wt} \%), \mathrm{ZnO}(0-8 \mathrm{wt} \%), \text { other oxides }(0-10 \mathrm{wt} \%), \text { coloring oxides }(0-8 \mathrm{wt} \%)\end{array}$ \\
\hline D-CAD & Dentin & CAD/CAM milling & Lithium disilicate ceramic (IPS e.max CAD; Ivoclar Vivadent, Schaan, Liechtenstein) \\
\hline D-PRE & & Heat-pressing & Lithium disilicate ceramic (IPS e.max Press; Ivoclar Vivadent): \\
\hline
\end{tabular}


Table 2 - The forces required to crack the material $\left(F_{\text {initial }}\right)$ and the load-bearing capacity $\left(F_{\max }\right)$ in Newton for all groups under investigation with mean, standard deviation (SD), first quartile (Q1), median, third quartile (Q3), minimum (min) and maximum (max).

\begin{tabular}{|l|l|r|r|r|r|r|}
\hline \multirow{2}{*}{ Group } & \multicolumn{9}{c|}{ Mean \pm SD } & Q1 & \multicolumn{2}{c|}{ Median Q3 } & Range min to max \\
\hline \multirow{2}{*}{ E-CAD } & $F_{\text {initial }}$ & $992 \pm 623$ & 600 & 845 & 1500 & 50 to 2300 \\
\cline { 2 - 7 } & $F_{\max }$ & $1555 \pm 759$ & 932 & 1495 & 2318 & 176 to 2494 \\
\hline \multirow{2}{*}{ E-PRE } & $F_{\text {initial }}$ & $837 \pm 372$ & 610 & 700 & 1050 & 300 to 1700 \\
\cline { 2 - 7 } & $F_{\max }$ & $1562 \pm 310$ & 1314 & 1575 & 1668 & 1009 to 2286 \\
\hline \multirow{2}{*}{ D-CAD } & $F_{\text {initial }}$ & $1180 \pm 454$ & 900 & 1400 & 1500 & 400 to 1700 \\
\cline { 2 - 7 } & $F_{\max }$ & $1800 \pm 442$ & 1555 & 1856 & 2013 & 480 to 2490 \\
\hline \multirow{2}{*}{ D-PRE } & $F_{\text {initial }}$ & $1215 \pm 457$ & 900 & 1100 & 1650 & 300 to 1900 \\
\cline { 2 - 7 } & $F_{\text {max }}$ & $1861 \pm 364$ & 1566 & 1877 & 2131 & 1139 to 2446 \\
\hline
\end{tabular}


Table 3 - The marginal and internal adaption in $\mu \mathrm{m}$ for all groups under investigation with mean, standard deviation (SD), first quartile (Q1), median, third quartile (Q3), minimum (min) and maximum (max) at the different locations "margin", "cusp", "fossa", as well as the 3D-adaption measurements ("color map"). Significant differences were detected in the area of the cusp (dashed line), fossa (dotted line) color map (continuous line).

\begin{tabular}{|c|c|c|c|c|c|c|c|c|c|c|c|c|c|}
\hline Group & Location & Mean \pm SD & Q1 & Median & Q3 & $\begin{array}{l}\text { Range min to } \\
\max \end{array}$ & \multicolumn{6}{|c|}{ Significances } & \\
\hline \multirow[t]{4}{*}{ E-CAD } & Margin & $127 \pm 57$ & 83 & 115 & 167 & 50 to 281 & \multirow{4}{*}{\multicolumn{2}{|c|}{$\begin{array}{ll}T & T \\
1 & 1 \\
1 & 1\end{array}$}} & & & & \\
\hline & Cusp & $127 \pm 57$ & 177 & 229 & 285 & 142 to 441 & & & & & & & \\
\hline & Fossa & $635 \pm 270$ & 451 & 560 & 729 & 351 to 1448 & & & & & & & \\
\hline & Color map & $216 \pm 67$ & 159 & 196 & 280 & 147 to 375 & & & & & & & \\
\hline \multirow[t]{4}{*}{ E-PRE } & Margin & $115 \pm 40$ & 90 & 113 & 137 & 55 to 192 & \multirow{4}{*}{\multicolumn{2}{|c|}{$\begin{array}{ll}1 & 1 \\
1 & 1 \\
1 & 1 \\
1 & 1 \\
1 & 1 \\
1 & 1\end{array}$}} & & \multirow{4}{*}{$\begin{array}{l}T \\
1 \\
1\end{array}$} & \multirow{4}{*}{\multicolumn{2}{|c|}{$\bar{\vdots} \quad \bar{\vdots}$}} & \multirow{4}{*}{$T$} \\
\hline & Cusp & $258 \pm 67$ & 205 & 239 & 315 & 177 to 404 & & & & & & & \\
\hline & Fossa & $663 \pm 155$ & 541 & 628 & 796 & 370 to 973 & & & & & & & \\
\hline & Color map & $212 \pm 38$ & 171 & 214 & 248 & 150 to 264 & & & & & & & \\
\hline \multirow[t]{4}{*}{ D-CAD } & Margin & $109 \pm 29$ & 86 & 107 & 118 & 72 to 181 & \multirow{4}{*}{\multicolumn{2}{|c|}{$\begin{array}{cc}1 & 1 \\
1 & 1 \\
1 & 1 \\
& 1\end{array}$}} & \multirow{4}{*}{$\begin{array}{l}1 \\
1 \\
1\end{array}$} & \multirow{4}{*}{$\begin{array}{l}1 \\
1 \\
1 \\
1 \\
1\end{array}$} & \multirow{4}{*}{$\begin{array}{l}\vdots \\
\vdots \\
\vdots \\
\vdots \\
\vdots\end{array}$} & \multirow{4}{*}{$\begin{array}{c}\vdots \\
\vdots \\
\vdots \\
\vdots\end{array}$} & \\
\hline & Cusp & $180 \pm 38$ & 151 & 185 & 211 & 120 to 252 & & & & & & & \\
\hline & Fossa & $287 \pm 106$ & 225 & 264 & 334 & 95 to 539 & & & & & & & \\
\hline & Color map & $176 \pm 34$ & 149 & 186 & 200 & 114 to 232 & & & & & & & \\
\hline \multirow[t]{4}{*}{ D-PRE } & Margin & $99 \pm 36$ & 75 & 93 & 120 & 49 to 188 & \multirow{4}{*}{\multicolumn{2}{|c|}{$\begin{array}{l}1 \\
1 \\
1\end{array}$}} & \multirow{4}{*}{\multicolumn{2}{|c|}{$\begin{array}{l}1 \\
1 \\
1\end{array}$}} & \multirow{4}{*}{\multicolumn{2}{|c|}{$\vdots$}} & \multirow{4}{*}{1} \\
\hline & Cusp & $145 \pm 55$ & 105 & 145 & 177 & 47 to 261 & & & & & & & \\
\hline & Fossa & $397 \pm 228$ & 162 & 406 & 580 & 66 to 798 & & & & & & & \\
\hline & Color map & $156 \pm 42$ & 120 & 155 & 187 & 82 to 238 & & & & & & & \\
\hline
\end{tabular}

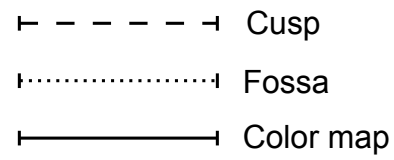


Table 4 - Failure types with percentage of no visible fracture (score 0), cohesive fracture within the restoration (score 1), cohesive fracture of the restoration and of the cement layer (score 2) and fracture of the restoration-cement-tooth complex (score 3).

\begin{tabular}{|c|c|c|c|c|}
\hline Group & Score 0 [\%] & Score 1 [\%] & Score 2 [\%] & Score $3[\%]$ \\
\hline E-CAD & 5 & 15 & 75 & 5 \\
\hline E-PRE & 5 & 0 & 95 & 0 \\
\hline D-CAD & 0 & 0 & 100 & 0 \\
\hline D-PRE & 5 & 0 & 95 & 0 \\
\hline
\end{tabular}




\section{Figures}

Figure 1 - Schematic drawing of the embedded specimen with the preparations within enamel (ECAD and E-PRE) or with exposed dentin (D-CAD and D-PRE). The restorations consisted of $0.5 \mathrm{~mm}$ thick occlusal veneers made out of lithium disilicate with different fabrication techniques (E-CAD and D-CAD: milling; E-PRE and D-PRE: heat-pressing).

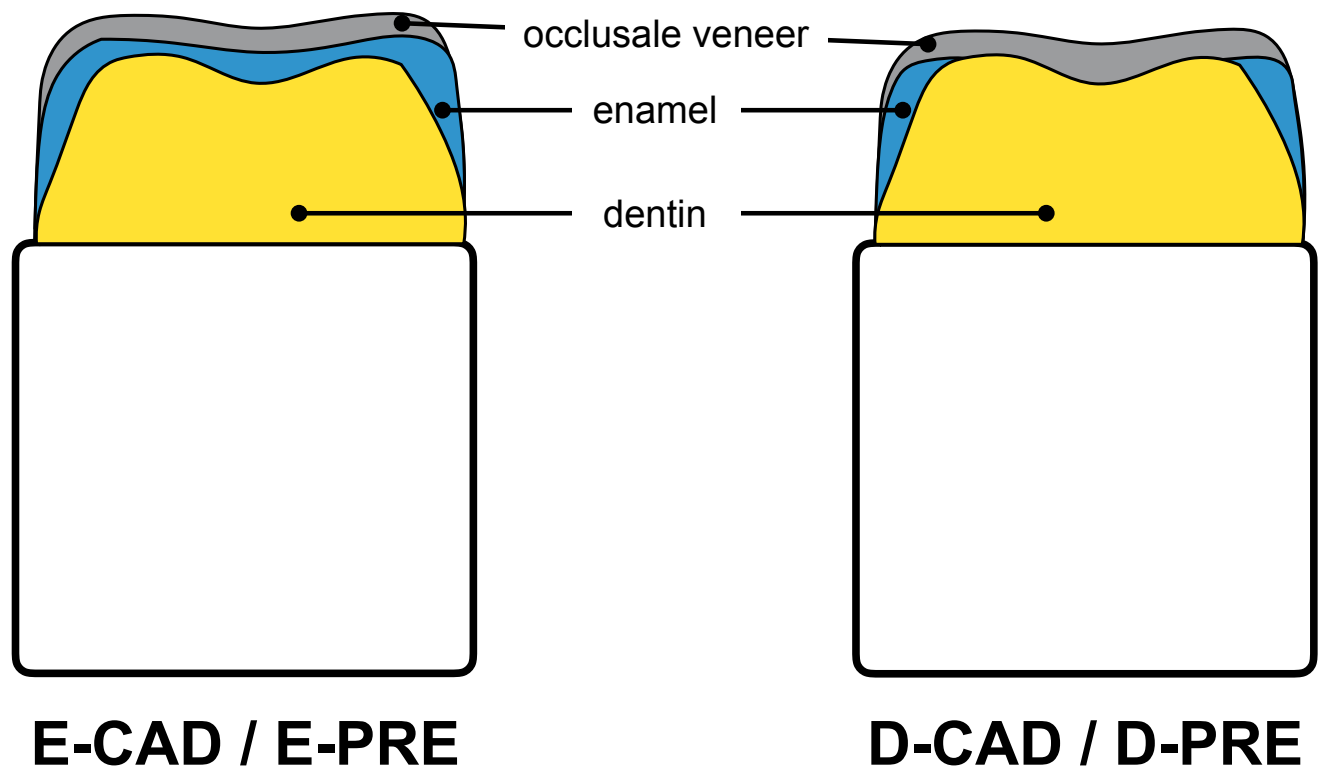


Figure 2 - Cross section for the measurement of the marginal and internal adaption. In mesio-distal (M-D) and disto-lingual (D-L) direction the measurements areas were at the margins, the cusps and the fossae.
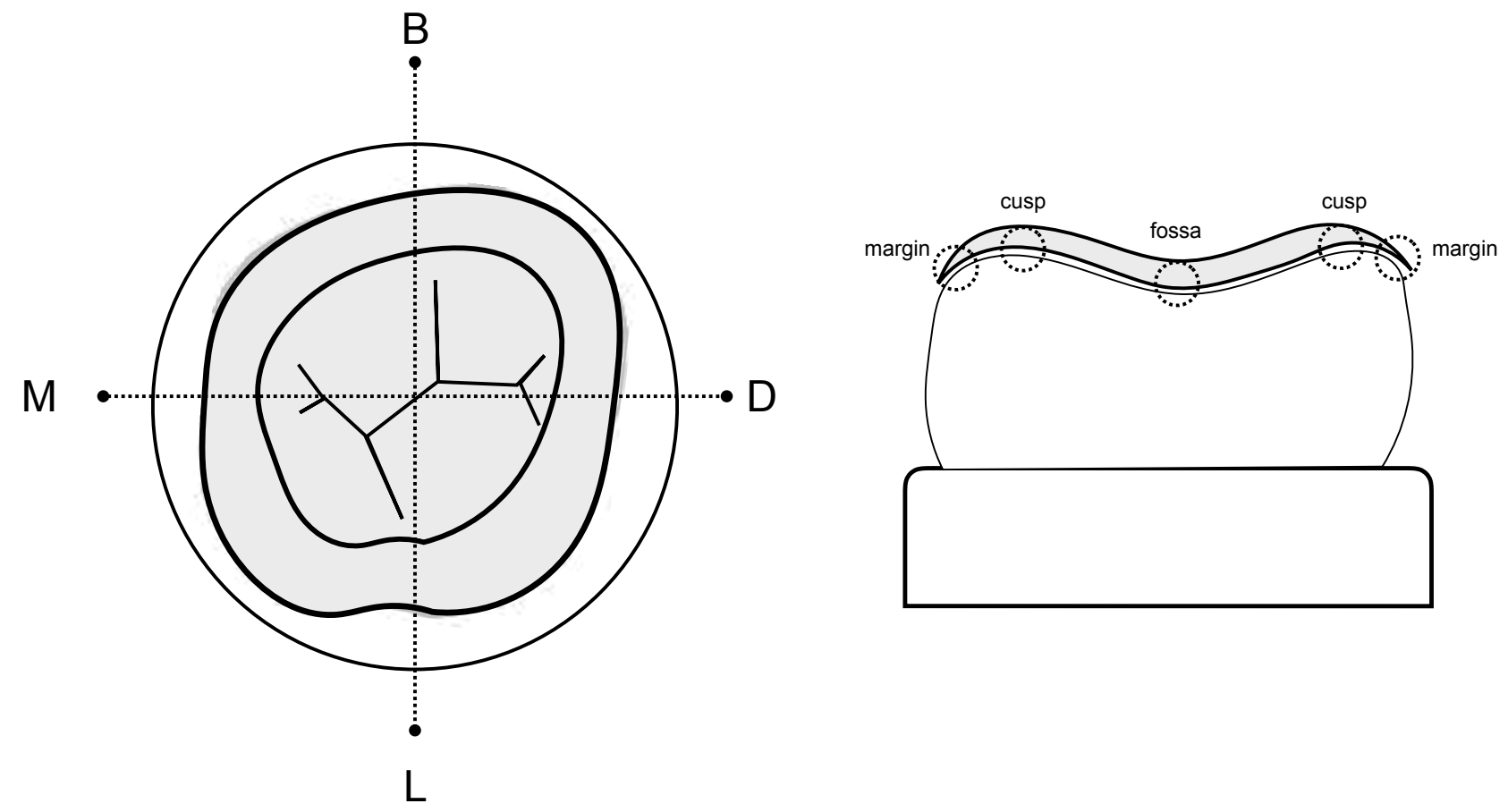
Figure 3 - Box-plots with the $F_{\text {initial }}$ values.

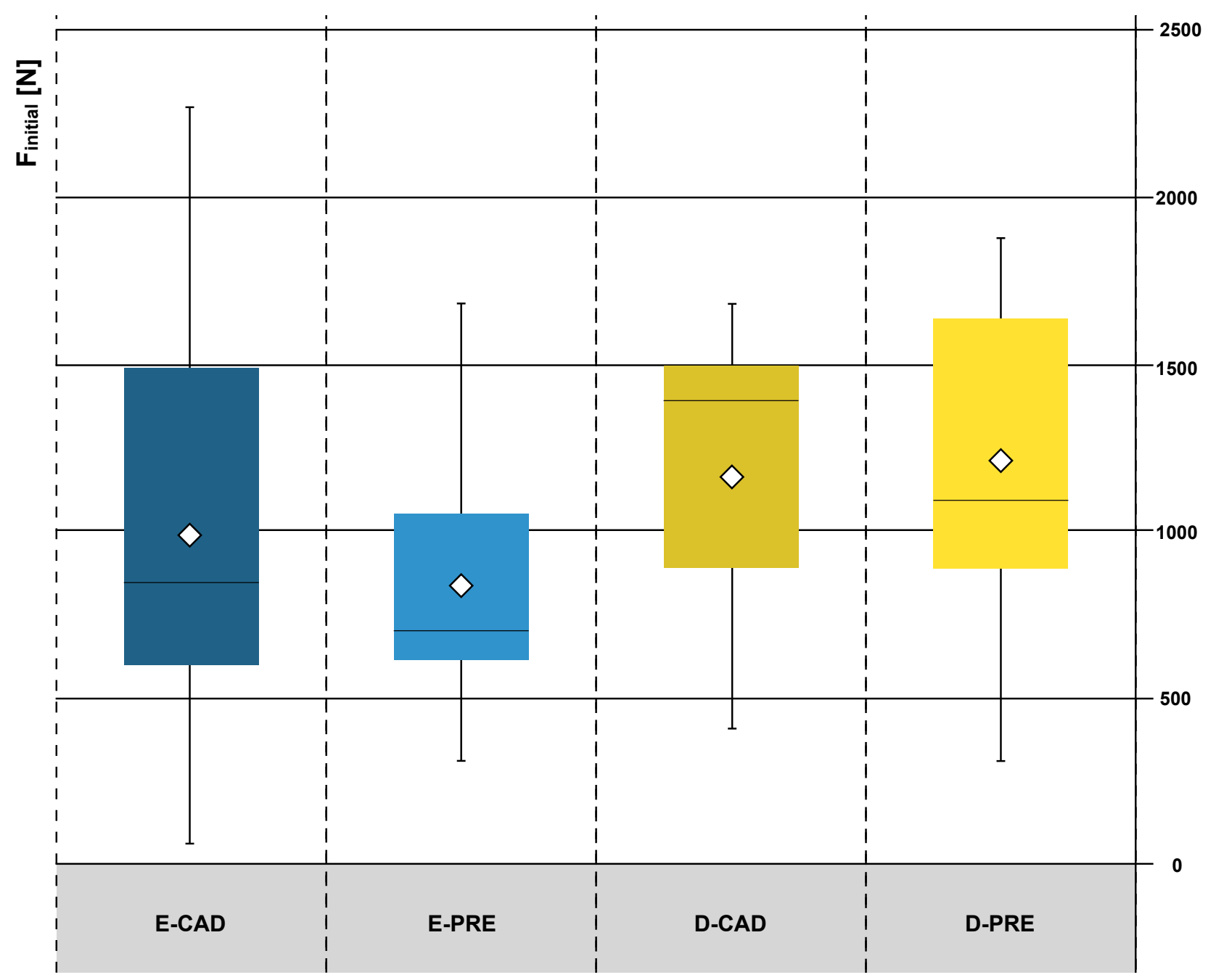


Figure 4 - Box-plots with the $F_{\max }$ values.

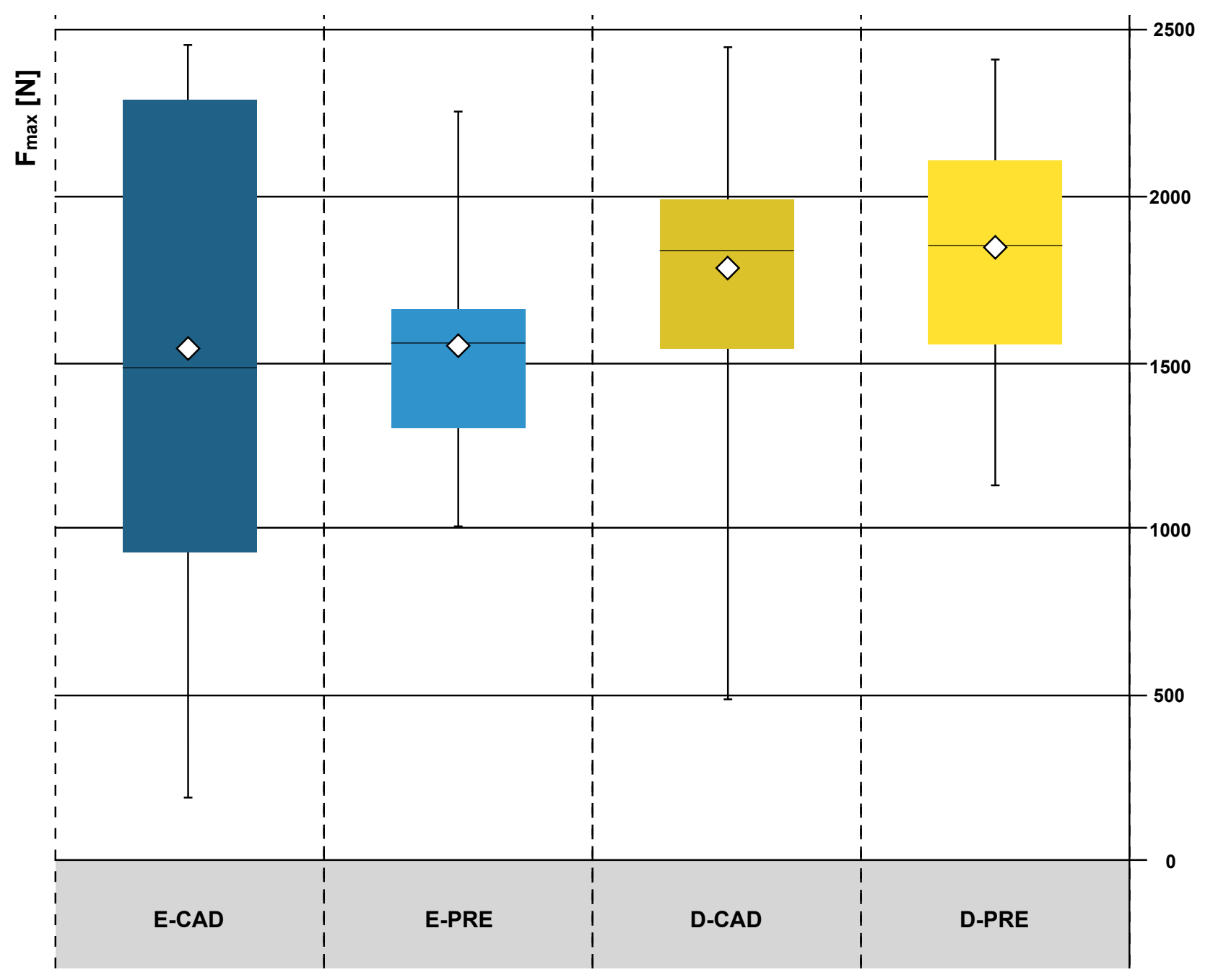


Figure 5 - Fit plot for $F_{\max }$ and the internal adaption (color map) of all groups pooled.

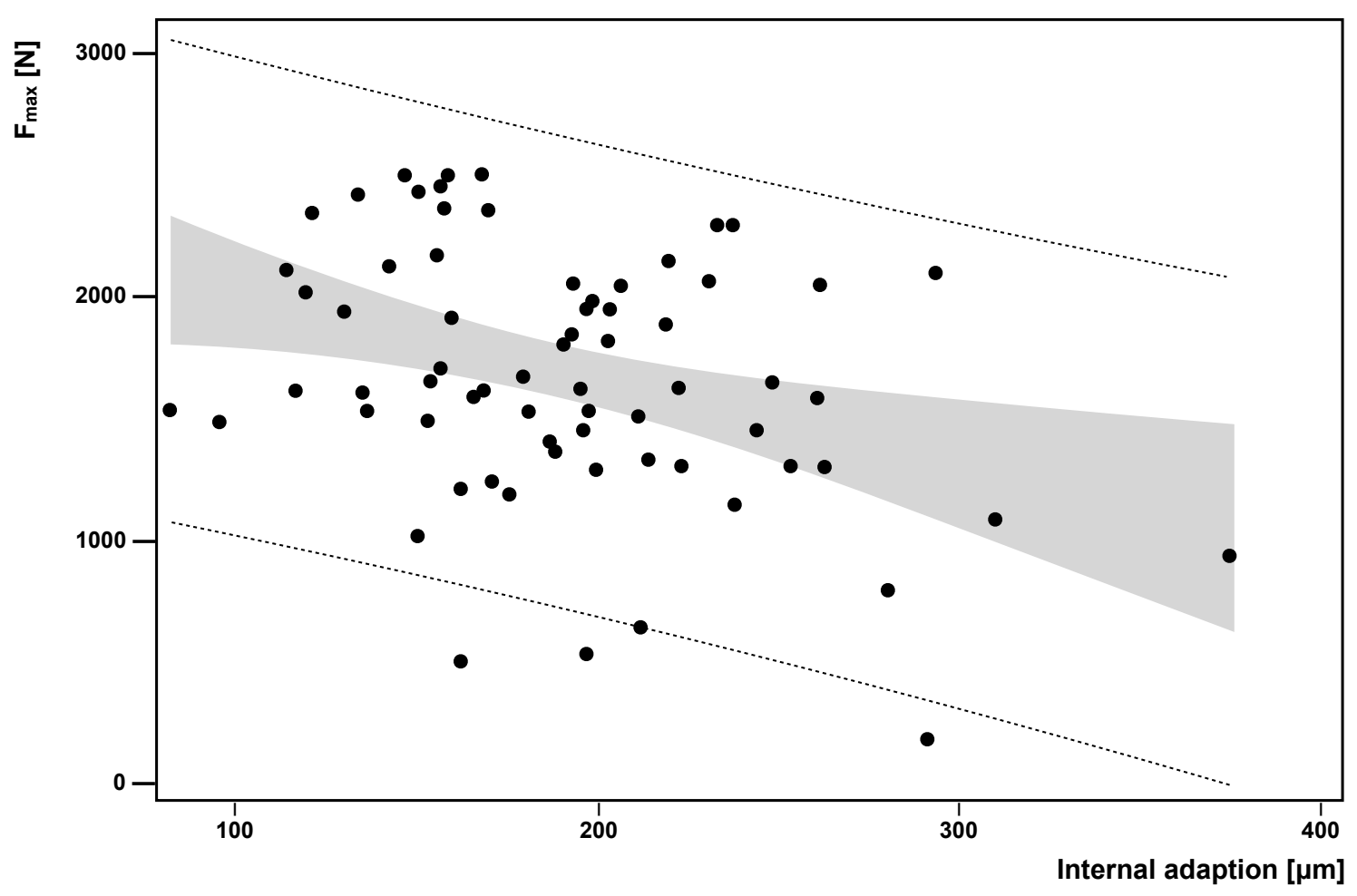




\section{References}

1. Loomans B, Opdam N, Attin T, Bartlett D, Edelhoff D, Frankenberger R, et al. Severe Tooth Wear: European Consensus Statement on Management Guidelines. J Adhes Dent 2017;19:111-119.

2. Lawson NC, Bansal R, Burgess JO. Wear, strength, modulus and hardness of CAD/CAM restorative materials. Dent Mater 2016;32:e275-e283.

3. Guazzato M, Albakry M, Ringer SP, Swain MV. Strength, fracture toughness and microstructure of a selection of all-ceramic materials. Part I. Pressable and alumina glass-infiltrated ceramics. Dent Mater 2004;20:441-448.

4. Guazzato M, Albakry M, Ringer SP, Swain MV. Strength, fracture toughness and microstructure of a selection of all-ceramic materials. Part II. Zirconia-based dental ceramics. Dent Mater 2004;20:449-456.

5. Krummel A, Garling A, Sasse M, Kern M. Influence of bonding surface and bonding methods on the fracture resistance and survival rate of full-coverage occlusal veneers made from lithium disilicate ceramic after cyclic loading. Dent Mater 2019;35:1351-1359.

6. Ioannidis A, Mühlemann S, Özcan M, Hüsler J, Hämmerle CH, Benic G. Ultra-thin occlusal veneers bonded to enamel and made of ceramic or hybrid materials exhibit load-bearing capacities not different from conventional restorations. Journal of the mechanical behavior of biomedical materials 2019:433-440.

7. Maeder M, Pasic P, Ender A, Özcan M, Benic G, loannidis A. Load-bearing capacities of ultra-thin occlusal veneers bonded to dentin. Journal of the mechanical behavior of biomedical materials 2019;95:165-171.

8. Ioannidis A, Bomze D, Hammerle CHF, Husler J, Birrer O, Muhlemann S. Load-bearing capacity of CAD/CAM 3D-printed zirconia, CAD/CAM milled zirconia, and heat-pressed lithium disilicate ultra-thin occlusal veneers on molars. Dent Mater 2020.

9. Alkadi L, Ruse ND. Fracture toughness of two lithium disilicate dental glass ceramics. J Prosthet Dent 2016;116:591-596.

10. Aboushelib MN, Elmahy WA, Ghazy MH. Internal adaptation, marginal accuracy and microleakage of a pressable versus a machinable ceramic laminate veneers. J Dent 2012;40:670677.

11. Oliva RA, Lowe JA, Ozaki MM. Film thickness measurements of a paint-on die spacer. J Prosthet Dent 1988;60:180-184. 
12. Zhang Y, Kim JW, Bhowmick S, Thompson VP, Rekow ED. Competition of fracture mechanisms in monolithic dental ceramics: flat model systems. Journal of biomedical materials research Part B, Applied biomaterials 2009;88:402-411.

13. Deng Y, Miranda P, Pajares A, Guiberteau F, Lawn BR. Fracture of ceramic/ceramic/polymer trilayers for biomechanical applications. Journal of biomedical materials research Part $A$ 2003;67:828-833.

14. Coelho PG, Silva NR, Bonfante EA, Guess PC, Rekow ED, Thompson VP. Fatigue testing of two porcelain-zirconia all-ceramic crown systems. Dent Mater 2009;25:1122-1127.

15. Park JM, Hammerle CHF, Benic GI. Digital technique for in vivo assessment of internal and marginal fit of fixed dental prostheses. J Prosthet Dent 2017;118:452-454.

16. Dahl BE, Ronold HJ, Dahl JE. Internal fit of single crowns produced by CAD-CAM and lostwax metal casting technique assessed by the triple-scan protocol. J Prosthet Dent 2017;117:400404.

17. Holst S, Karl M, Wichmann M, Matta RE. A new triple-scan protocol for 3D fit assessment of dental restorations. Quintessence Int 2011;42:651-657.

18. Krejci I, Reich T, Lutz F, Albertoni M. [An in vitro test procedure for evaluating dental restoration systems. 1. A computer-controlled mastication simulator]. Schweiz Monatsschr Zahnmed 1990;100:953-960.

19. Bates JF, Stafford GD, Harrison A. Masticatory function--a review of the literature. 1. The form of the masticatory cycle. J Oral Rehabil 1975;2:281-301.

20. DeLong R, Douglas WH. An artificial oral environment for testing dental materials. IEEE Trans Biomed Eng 1991;38:339-345.

21. Steiner M, Mitsias ME, Ludwig K, Kern M. In vitro evaluation of a mechanical testing chewing simulator. Dent Mater 2009;25:494-499.

22. Silva NR, de Souza GM, Coelho PG, Stappert CF, Clark EA, Rekow ED, et al. Effect of water storage time and composite cement thickness on fatigue of a glass-ceramic trilayer system. Journal of biomedical materials research Part B, Applied biomaterials 2008;84:117-123.

23. Mously HA, Finkelman M, Zandparsa R, Hirayama H. Marginal and internal adaptation of ceramic crown restorations fabricated with CAD/CAM technology and the heat-press technique. J Prosthet Dent 2014;112:249-256. 
24. Guess PC, Vagkopoulou T, Zhang Y, Wolkewitz M, Strub JR. Marginal and internal fit of heat pressed versus CAD/CAM fabricated all-ceramic onlays after exposure to thermo-mechanical fatigue. J Dent 2014;42:199-209.

25. Zeltner M, Sailer I, Muhlemann S, Ozcan M, Hammerle CH, Benic GI. Randomized controlled within-subject evaluation of digital and conventional workflows for the fabrication of lithium disilicate single crowns. Part III: marginal and internal fit. J Prosthet Dent 2017;117:354-362.

26. Benic GI, Sailer I, Zeltner M, Gutermann JN, Ozcan M, Muhlemann S. Randomized controlled clinical trial of digital and conventional workflows for the fabrication of zirconia-ceramic fixed partial dentures. Part III: Marginal and internal fit. J Prosthet Dent 2019;121:426-431.

27. Clausen JO, Abou Tara M, Kern M. Dynamic fatigue and fracture resistance of non-retentive all-ceramic full-coverage molar restorations. Influence of ceramic material and preparation design. Dent Mater 2010;26:533-538.

28. Ender A, Attin T, Mehl A. In vivo precision of conventional and digital methods of obtaining complete-arch dental impressions. J Prosthet Dent 2016;115:313-320.

29. Ender A, Mehl A. Accuracy of complete-arch dental impressions: a new method of measuring trueness and precision. J Prosthet Dent 2013;109:121-128.

30. Marghalani A, Weber HP, Finkelman M, Kudara Y, El Rafie K, Papaspyridakos P. Digital versus conventional implant impressions for partially edentulous arches: An evaluation of accuracy. J Prosthet Dent 2018;119:574-579.

31. Papaspyridakos P, Gallucci GO, Chen CJ, Hanssen S, Naert I, Vandenberghe B. Digital versus conventional implant impressions for edentulous patients: accuracy outcomes. Clin Oral Implants Res 2016;27:465-472.

32. Segerstrom S, Wiking-Lima de Faria J, Braian M, Ameri A, Ahlgren C. A Validation Study of the Impression Replica Technique. J Prosthodont 2019;28:e609-e616.

33. Scholtanus JD, Ozcan M. Clinical longevity of extensive direct composite restorations in amalgam replacement: up to 3.5 years follow-up. J Dent 2014;42:1404-1410. 